\title{
Thermography measurement of the local heat transfer distribution for flow around a surface-mounted obstacle
}

bY LYBAERT P., FELDHEIM V. and LEBRUN I.

\author{
Laboratoire de Thermique, Faculté Polytechnique de Mons, 56, rue de I'Epargne, \\ B-7000 Mons, Belgium.
}

\begin{abstract}
This paper deals with the use of infrared thermography to measure the convective heat transfer coefficient distribution at the surface of a plate around a surface-mounted prismatic obstacle, placed in a turbulent flow. Complex longitudinal profiles of the coefficient are obtained, showing minimum values upstream from the obstacle, minimum and maximum values downstream. The heat transfer data are analyzed with recent results from the literature concerning the fluid flow around the obstacle. The measured values are then compared with values obtained by numerical simulation, using the standard $(k, \varepsilon)$ model of turbulence. The comparison shows that the model overpredicts the reattachment length and underpredicts the heat transfer coefficients.
\end{abstract}

\section{Nomenclature}

d obstacle height $(\mathrm{m})$

$h \quad$ heat transfer coefficient $\left(W \cdot m^{-2} \cdot K^{-1}\right)$

$k \quad$ thermal conductivity $\left(W \cdot m^{-1} \cdot K^{-1}\right)$

f black body pixel intensity (I.U.)

$I^{\prime} \quad$ measured pixel intensity (I.U.)

I obstacle length (m)

$\mathrm{Nu} \quad$ Nusselt number $(h d / k)$

Re Reynolds number $(U d / v)$

$t \quad$ support plate thickness $(\mathrm{m})$

$T$ temperature (K)

$U$ fluid velocity $\left(\mathrm{m} . \mathrm{s}^{-1}\right)$

$x \quad$ longitudinal coordinate $(m)$

$y$ transverse coordinate $(\mathrm{m})$

$w \quad$ obstacle width $(\mathrm{m})$

$\begin{array}{ll}\text { Greek } & \text { symbols } \\ \delta & \text { boundary layer thickness (m) } \\ \varepsilon & \text { emission factor } \\ \phi & \text { heat flux }\left(W \cdot \mathrm{m}^{-2}\right) \\ \sigma & \text { Stefan Boltzmann constant } \\ \tau & \text { transmission factor } \\ \text { Subscripts } \\ a & \text { air } \\ d & \text { at flow detachment } \\ e l & \text { due to Joule dissipation } \\ \text { loss } & \text { lost to the environment } \\ o & \text { heated plate (object) } \\ r & \text { at flow reattachment } \\ \text { wa } & \text { tunnel walls } \\ \text { wi } & \text { window }\end{array}$

\section{Introduction}

Numerous experimental and numerical studies have dealt with separated flow and heat transfer around obstacles during the last two decades. Besides their interest for industrial applications, these configurations are often used as benchmark situations to validate the numerical models of turbulent fluid flow and convective heat transfer.

This paper deals with heat transfer at the surface of a plate with a surface-mounted prismatic obstacle, placed in a parallel turbulent fluid flow. This flow configuration is the 3-D analogie of the much more documented 2-D fluid flow around a surface-mounted rectangular cylinder.

Data about the flow have been published in several recent papers [1-3]. In the two* dimensional case (rectangular cylinder), the flow separates from the wall upstream of the 
http://dx.doi.org/10.21611/qirt.1994.028

obstacle and reattaches downstream, giving vortical regions on both sides of the rib. The shape of the perturbed zone and the positions of the detachment and reattachment points depend on many factors, in particular on the obstacle aspect ratio (length-to-height ratio $\| / d n$, the flow velocity (Reynolds number), and the relative boundary layer thickness ( $\delta / d$ ratio).

The flow structure is much more complicated in the three-dimensional situation (firure 1). In this case, the fluid flows around as well as over the obstacle. This leads to shorter detachment and reattachment lengths than in 2-D. Furthermore in 3-D, these lengths are also functions of the obstacle width (width-to-height ratio $w / d$ ).

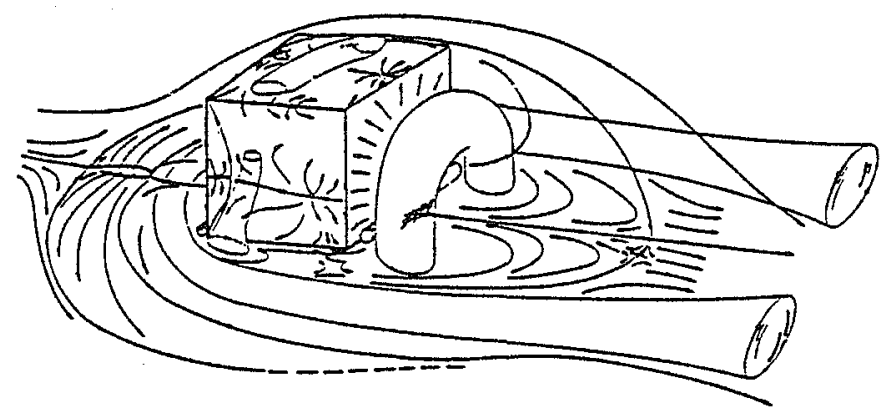

Fig.1 - Configuration of the flow around a surface-mounted obstacle [2]

Heat transfer data are rather sparse in the literature and, to our knowledge, published material only concerns the two-dimensional flow situation [4-5].

\section{Experimental method}

The experimental method has been presented in a previous paper [6]. The local heat transfer coefficient distribution is obtained from the steady-state measurement of the wall temperature field by means of an infrared scanner.

\subsection{Experimental facility}

The experimental setup (figure 2) uses a small open loop wind tunnel. Ambient air is pushed through the tunnel by a variable speed blower, flows through a pocket filter and a 18:1 contraction before entering the test section. The latter has a rectangular cross section of $0.2 \times 0.1 \mathrm{~m}^{2}$ (width $x$ height) and a length of $1.5 \mathrm{~m}$. Air velocity can be varied from 1 to $15 \mathrm{~m} / \mathrm{s}$, giving Reynolds numbers based on the section height ranging from $10^{4}$ to $10^{5}$.

The plate and obstacle geometry is represented in figure 3 . The plate is a printed circuit board fitted on the wall of the tunnel and backed by a $25 \mathrm{~mm}$ thick polyurethane insulating layer. A uniform heat flux at the surface of the plate is obtained by Joule dissipation. The board consists of a stiff epoxy sheet, of $1.6 \mathrm{~mm}$ thickness, coated with a $34 \mu \mathrm{m}$ thick copper layer. The required electrical resistance is obtained by a zig-zag circuit of equally spaced transversal strips of $2.15 \mathrm{~mm}$ width, with $0.35 \mathrm{~mm}$ gap between them. The surface is painted black with high emissivity enamel. The obstacle is a thermally passive plexiglas parallelepiped. Its height $d$ is $20 \mathrm{~mm}$, its width $w$ and length $/$ are $40 \mathrm{~mm}$ 


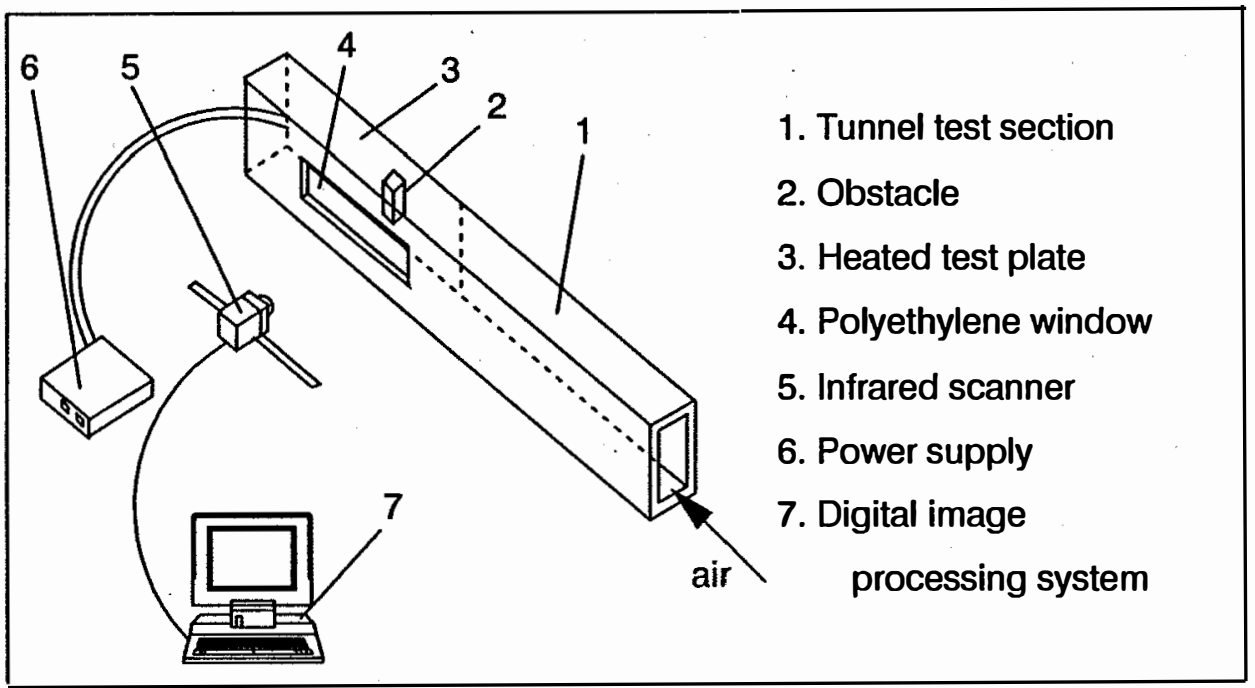

Fig.2 - Experimental facility
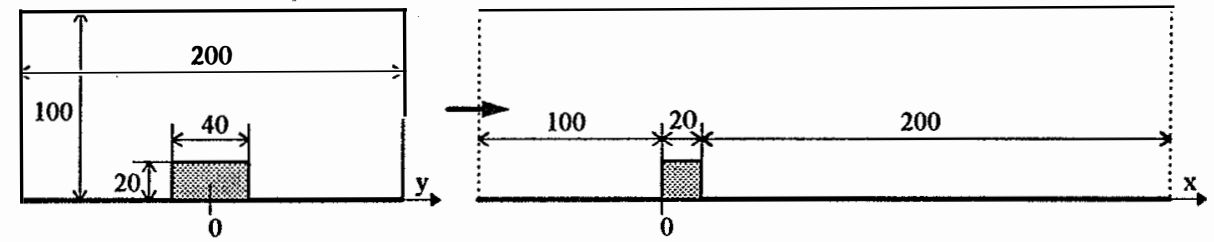

\section{Fig.3 - Plate and obstacle geometry}

The plate surface temperature field is measured by an AGEMA Thermovision $880 \mathrm{LW}$ system, fitted out with a $12^{\circ}$ lens. The system has a $\mathrm{HgCdTe}$ detector cooled by liquid nitrogen and operating in the 8-12 $\mu \mathrm{m}$ range. The AGEMA CATSE image processing package is used to digitize the video signal. The package produces $140 \times 140$ pixels infrared pictures with pixel intensity being coded in a 8-bit format. The pictures are stored on disk for further processing. The plate temperature is also measured by 10 thermocouples placed along the axis of the surface viewed by the scanner. These measurements are used for in-situ calibration of the infrared measuring system.

The surface of the plate is viewed by the IR scanner through a polyethylene film window. The film is standard plastic film from $3 \mathrm{M}$ used for do-it-yourself double-glazing. Its thickness is about $25 \mu \mathrm{m}$. For measurements in atmospheric pressure low velocity wind tunnels, polyethylene film is a very cheap alternative to expensive germanium or zinc selenide windows. However, its reflectance (about 0.09 ) is higher and special procedures are required in order to get accurate temperature measurements. 


\subsection{Measurement methods}

In order to eliminate the effect of parasitic radiation sources, the temperature field is measured by a relative method [6], which is based on the subtraction of two pictures: the first one is taken with the plate heated, the second one is taken while blowing air on the cold plate, i.e. with no heat flux at the surface, both pictures being taken at steady-state. By applying the radiometric equation to both experiments and subtracting the second equation from the first, and provided radiation from the wall and from the external environment did not change during the experiments, their contributions cancel out in the subtraction and the surface temperature during the first experiment is given by

$$
I(T)=I\left(T_{a}\right)+\frac{l^{\prime}-l(\varphi=0)}{\tau_{w 1} \varepsilon_{0}}
$$

In this equation, I(T) is the black body intensity at the plate temperature, from which the temperature $T$ can be obtained by applying the calibration equation of the scanner. As in the second experiment, the plate temperature is uniform and equal to the air temperature $T_{0}, I\left(T_{0}\right)$ can be taken equal to the calculated value of the black body intensity at $T_{\text {e. }} I^{\prime}$ and $I^{\prime}(\phi=0)$ are the measured intensities in the 'hot' and 'cold' experiments. $\tau_{w}$ is the transmission factor of the window and $\varepsilon_{0}$ is the plate emissivity.

The value of the product $\tau_{w} / \varepsilon$ is obtained by in-situ calibration, i.e. by fitting the temperatures measured by the scanner to the values measured by the thermocouples. The value which has been obtained by this procedure is about 0.85 .

The local heat transfer coefficient $h$ is related to the plate temperature by the energy balance written for a surface element, given by

$$
h\left(T-T_{a}\right)=\varphi_{\theta t}-\varphi_{\text {loss }}+\left(k_{x} \frac{\partial^{2} T}{\partial x^{2}}+k_{y} \frac{\partial^{2} T}{\partial y^{2}}\right) t-\varepsilon_{o} \sigma\left(T^{4}-T_{\text {wa }}^{4}\right)
$$

The terms of the R.H.S. of equation (2) are respectively the applied heat flux $\varphi_{0}$ due to Joule dissipation, the flux pross lost to the environment through the insulation at the back of the plate, the conduction heat flux exchang radiation heat flux between the plate surface and the walls of the tunnel, assumed to be black surfaces. $k_{x}$ and $k_{y}$ are the $x$ and $y$ direction thermal conductivities of the epoxy/copper composite, $t$ is the plate thickness.

\section{Experimental results}

The longitudinal profiles of the local convection coefficient obtained for an upstream air velocity of $10 \mathrm{~m} / \mathrm{s}$ are shown in figure 4 . The corresponding Reynolds number, based on the obstacle height $d$, is about 13,200. The different curves are given for different distances $y$ from the symmetry line of the plate. The following trends are observed:

- for $y / d<1$ : upstream from the obstacle, the heat transfer coefficient decreases, goes through a minimum at a distance $x$ of about $1.7 d$ from the obstacle and then increases. Behind the obstacle, the coefficient decreases, is minimum for $x / d=1.5$ (i.e. at a distance of $0.5 d$ from the rear face of the obstacle), then increases, has a maximum value for $x / d=3.5$ and then decreases continuously.

- for $y / d>1$ : from the leading edge of the plate, the coefficient decreases, goes through a minimum, then increases, goes through a maximum and then decreases continuously. Heat transfer at the surface of the plate is influenced by the obstacle up to a distance $y=2.5 d$ from the symmetry line. 
The shape of these profiles results from the structure of the flow around the otstacle. The flow around a surface-mounted cube is sketched in figure 1. The same overall pattern is obtained for small aspect ratios parallelepiped obstacles, the relative dimensions of the different structures being dependent on the aspect ratio. The initial decrease of the convection coefficient, upstream of the obstacle, is due to the development of the thermal boundary layer. The steep increase of the coefficient in front of the obstacle is; more difficult to analyze. It is partly caused by a local cooling effect, due to the thirmally passive obstacle which acts as a fin. However, a numerical evaluation shows that, for a heat transfer coefficient of $50 \mathrm{~W} \cdot \mathrm{m}^{-2} \cdot \mathrm{K}^{-1}$, the error on the coefficient resulting from this cooling effect has a maximum value of about $30 \%$ at the base of the obstacile and becomes negligibly small $10 \mathrm{~mm}$ upstream. Hence, the increase of the coefficient is due to the heat transfer with the horseshoe vortex which develops in front of the obstacle. The minimal values of the coefficient are obtained where the flow separates from the plate.

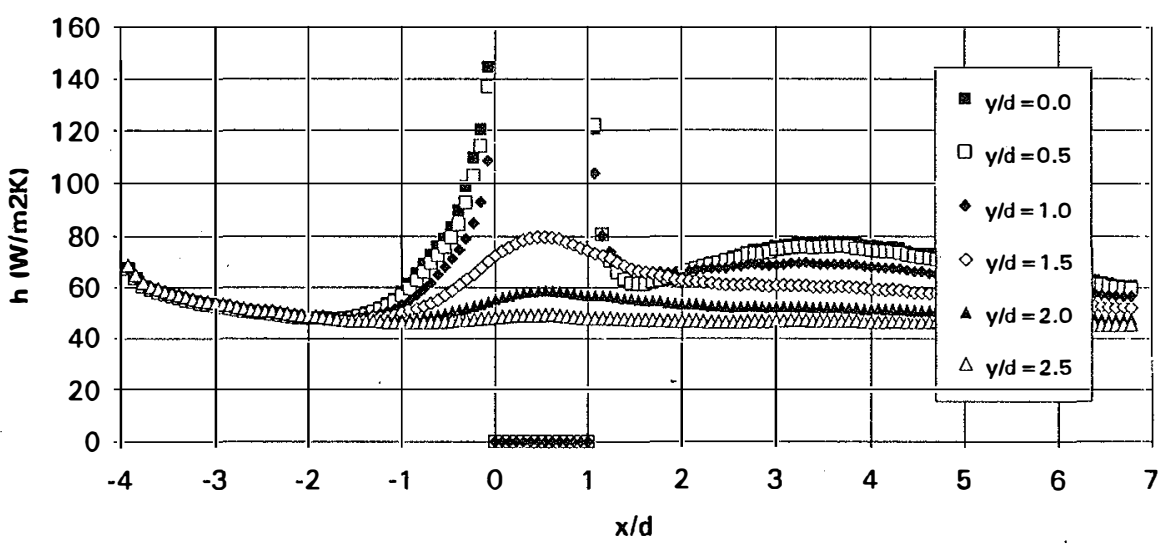

Fig.4 - Longitudinal profiles of the local heat transfer coefficient $U=10 \mathrm{~m} / \mathrm{s}, \operatorname{Re}=13,200$

The profiles obtained downstream of the obstacle are similar to those obtained for twodimensional flows [4,5]. Maximum heat transfer coefficients are obtained where the flow reattaches the plate, lower coefficients are observed downstream of the obstacle where the plate is in contact with a "bubble " of recirculating fluid. The high coefficient values measured just behind the obstacle are probably due to a small secondary vortex which develops at the junction of the obstacle and the plate. A similar vortex has been observed by Buchlin [4] for plane flow around a rectangular cylinder.

The profiles of the local Nusselt numbers, measured along the symmetry line of the plate for different Reynolds number values $(R e=U d / \nu)$, are represented in figure 5 . The figure shows that the maxima and minima are more pronounced at higher Reynolds numbers. The position of the reattachment point on the axis lassumed to be located at the position of the maximum coefficient) is seen to be independent of the Reynolds number, for $R e$ values higher than 5,000. $x_{r} / d$ is then equal to about 3.5. For lower $R e$ values, the $\mathrm{Nu}$-profiles are so flat that the positions of the maximum $\mathrm{Nu}$ values are very difficult to detect. The position $x_{d}$ of the upstream minimum value depends on the Reynolds number: $x_{d} / d$ decreases from 2.3 to 1.5 when Re increases from 2,000 to 17,000 .

This variation should be due to the variation of the boundary layer thickness upstream of the obstacle with the Reynolds number. In our tests, the boundary layer is laminar at: the leading edge of the plate, for velocities up to $12 \mathrm{~m} / \mathrm{s}$. Its thickness decreases when the 
http://dx.doi.org/10.21611/qirt.1994.028

velocity increases, its calculated value ranges from 14 to $5 \mathrm{~mm}$ (i.e. $\delta / d$ ranging from 0.7 to 0.251 when the velocity varies from 1.5 to $12 \mathrm{~m} / \mathrm{s}$. Hence, the distance of the detachment point is seen to increase with the boundary layer thickness, a result which is consistent with the flow visualization data of Shofield and Logan [1]. On the other hand, the boundary layer thickness seems to have no effect on the downstream reattachment length.

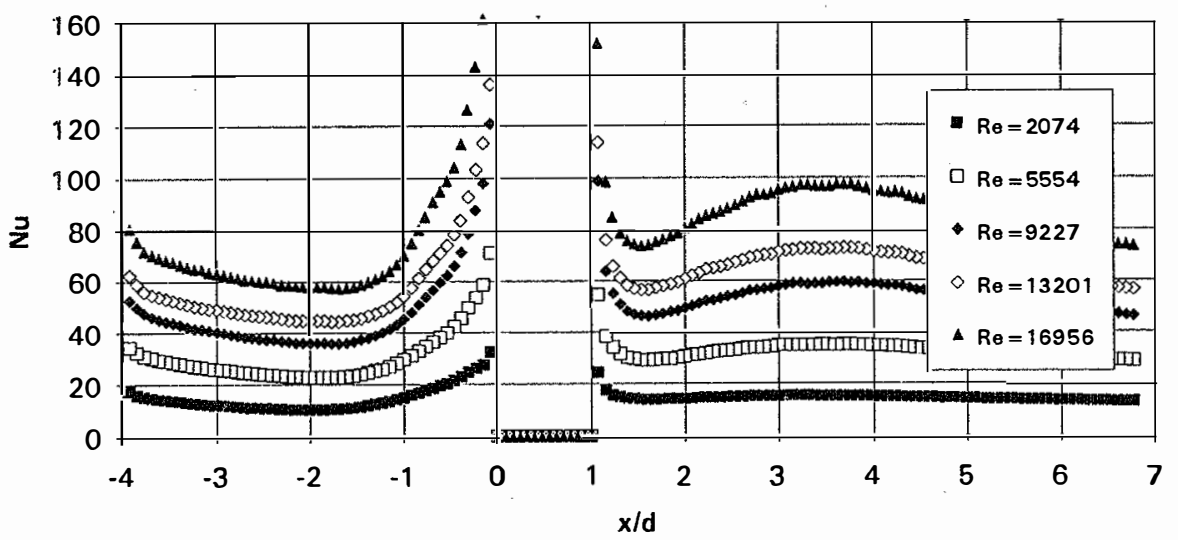

Fig.5.- Nusselt number profile along the symmetry line - Effect of the Reynolds number

The reattachment length we have measured is also identical to the value measured by flow visualization techniques by Martinuzzi and Tropea [2] for prismatic obstacles of the same aspect ratio in a fully developed turbulent flow. For the upstream detachment length, however, their measured value is about 1.2 times the obstacle height, i.e. lower than our values. This result is consistent with the assumption that the boundary layer thickness only influences the location of the upstream separation line.

\section{Numerical simulation}

The turbulent flow and convective heat transfer around the obstacle have been computed by the FLUENT code, version 4.22 . The classical two equation $(k, \varepsilon)$ turbulence model has been used, with the standard one-layer wall function approach to handle wall boundaries. The equations are solved by a finite volume method, using the same structured grid for all the variables.

The dimensions of the computational domain are $0.1 \times 0.1 \times 0.35 \mathrm{~m}^{3}$, with the inlet and outlet boundaries located $7.5 d(0.15 \mathrm{~m})$ upstream and $9 d(0.18 \mathrm{~m})$ downstream of the obstacle respectively. A grid of $22 \times 24 \times 82$ control volumes has been used in the computation. Figure 6 shows the velocity field obtained for an inlet velocity of $10 \mathrm{~m} / \mathrm{s}$. The predicted upstream separation distance is about $0.5 d$, i.e. about one third the measured value. The calculated downstream reattachment length, measured from the rear face of the prism, is 4.7 times the obstacle height, i.e. about two times the measured value of $2.5 \mathrm{~d}$. A single downstream vortex is obtained by the numerical computation, a finer grid is needed to predict the secondary vortex behind the obstacle.

As to the heat transfer, the model predicts minimum values of the coefficient along the separation line and maximal values at reattachment. As no secondary vortex is predicted, no minima are obtained downstream of the obstacle. Furthermore, the computed heat 
transfer coefficients are always lower than the measured ones and the variations are less pronounced.

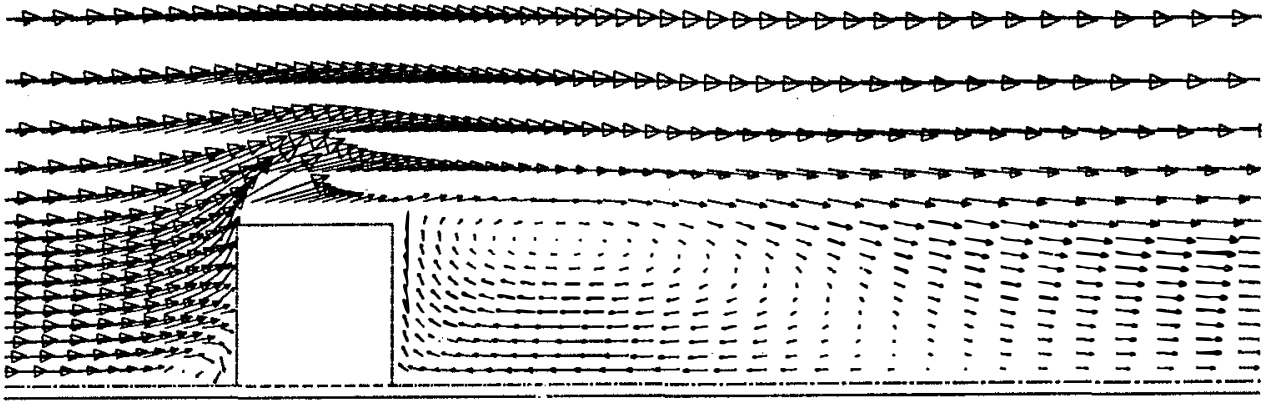

Fig.6 - Numerical simulation - Velocity field in the symmetry plane $U=10 \mathrm{~m} / \mathrm{s}, R e=13,200$

These discrepancies between calculated results and measured data have also been observed in two-dimensional situations [5,7]. They are due to the isotropy assumption of the $(k, \varepsilon)$ model and to the use of standard wall functions. A higher order turbulence model (e.g. RSM model) should improve the prediction of the flow. In order to compute heat transfer more accurately, a more sophisticated model has to be used near the walls. Higher order near-wall turbulence models (two- or three-layer wall functions [7]) or low Reynolds number models (damping functions) are needed.

\section{Conclusions}

The distribution of the local heat transfer coefficient at the surface of a plate around a surface-mounted prismatic obstacle (aspect ratios $1 / d=1, w / d=2$ ), placed in a parallel turbulent flow has been measured by infrared thermography. This 3-D flow with separation leads to a very complex distribution, with minimal coefficient values upstream of the obstacle and maximal values downstream. The shape of the distribution is due to upstream separation and downstream reattachment of the flow. For high Reynolds numbers, the reattachment length on the symmetry line of the plate has been shown to be independent of the Reynolds number. The measured length is about 2.5 times the obstacle height (measured from the rear face of the prism) and is consistent with literature data obtained by flow visualization experiments. The upstream separation length depends on the Reynolds number and the boundary layer thickness, additional experiments are needed to assess the effects of these variables.

Numerical modeling, based on classical turbulence models, overestimates the reattachment lengths and underestimates the heat transfer coefficients. Higher order turbulence models (RSM, RNG) and more sophisticated wall functions should be used to improve the accuracy of the predictions. 


\section{REFERENCES}

[1] SHOFIELD (W.H.), LOGAN (E.).- Turbulent shear flow over surface mounted obstacles. Journal of Fluids Engineering, 112, 376-385, (1990).

[2] MARTINUZZI (R.). TROPEA (C.). The flow around surface mounted prismatic obstacles placed in a fully developed channel flow. Journal of Fluids Engineering, 115, 85-92, (1993).

[3] HE (J.), SONG (C.S.). Computation of turbulent shear flow over surface-mounted obstacle. Journal of Engineering Mechanics, 118, 2282-2297, (1992).

[4] BUCHLIN (J.-M.). Heat transfer behind a rectangular cylinder in a boundary layer. Workshop on "Advanced Infrared Technology and Applications", Capri, (1993).

[5] ALIAGA (D.A.), LAMB (J.P.), KLEIN (D.E.). Convection heat transfer distributions over plates with square ribs from infrared thermography measurements. Int. J. Heat Mass Transfer, 37, 363-374, (1994)

[6] LYBAERT (P.), DURIEU (E.), SIRJACOBS (R.), FELDHEIM (V.). Measurement of local heat transfer coefficients in three-dimensional separated flow by thermography technique. First International Thermal Energy Congress Proceedings, ITEC-93, 6-10 June 1993, Marrakesh, 713-717, (1993).

[7] DJILALI (N.), GARTSHORE (I.), SALCUDEAN (M.), Calculation of convective heat transfer in recirculating turbulent flow using various near-wall turbulence models. Numer. Heat Transfer, Part A, 16, 189-212, (1989). 